\title{
Most primary central nervous system diffuse large B-cell lymphomas occurring in immunocompetent individuals belong to the nongerminal center subtype: a retrospective analysis of 31 cases
}

Eyas M Hattab ${ }^{1}$, Sarah E Martin ${ }^{1}$, Sohaib M Al-Khatib ${ }^{1}$, William J Kupsky ${ }^{2}$, Gail H Vance ${ }^{3}$, Ryan A Stohler ${ }^{3}$, Magdalena Czader ${ }^{1}$ and Mousa A Al-Abbadi ${ }^{4}$

${ }^{1}$ Department of Pathology and Laboratory Medicine, Indiana University School of Medicine, Indianapolis, IN, USA; ${ }^{2}$ Department of Pathology, Wayne State University, Detroit, MI, USA; ${ }^{3}$ Department of Medical and Molecular Genetics, Indiana University School of Medicine, Indianapolis, IN, USA and ${ }^{4}$ Department of Pathology, James H. Quillen Veterans Affairs Medical Center, Mountain Home, TN, USA

Primary central nervous system lymphomas are rare neoplasms characterized by a dismal prognosis relative to other extranodal lymphomas. Approximately $98 \%$ of primary central nervous system lymphomas are of B-cell origin, and most belong to the diffuse large B-cell type. Recently, diffuse large B-cell lymphomas have been subcategorized into germinal center and nongerminal center types based on gene expression profiles and immunohistochemical expression of $\mathrm{CD} 10, \mathrm{Bcl}-6$, and MUM1. Studies have shown that the overall survival rate of the germinal center group is better than that of the nongerminal center lymphomas. In this study, 31 cases of primary central nervous system lymphomas of the diffuse large B-cell type were retrieved, reviewed, and immunostained for CD10, Bcl-6, MUM1, and Ki-67. Subclassification was carried out as described earlier, where CD10 and/or Bcl-6 positivity and negativity for MUM1 were considered characteristic of germinal center subtype and the opposite expression of nongerminal center subtype. Furthermore, the proliferative activity was semiquantitatively assessed using percent positive cells staining with Ki-67. Of the 31 cases examined, 26 (84\%) were found to belong to the nongerminal center type. The Ki-67 index in these 26 cases ranged from 30 to $90 \%$ (mean, 69\%). Five cases were categorized as the germinal center subtype. They had an Ki-67 index between 70 and $90 \%$ (mean, $\mathbf{7 8 \%} \%$. Interestingly, none of our patients were known to be HIV positive. One patient had a 10-year history of orthotopic liver transplant. We also performed fluorescence in situ hybridization analysis on formalin-fixed material and found that $38 \%$ of the cases where tissue was available had abnormalities of $\mathrm{MYC} /$ $I G H$ and/or IGH/BCL2. We conclude that most primary central nervous system diffuse large B-cell lymphomas are of the nongerminal center origin. Regardless of the germinal center status, all cases showed a high proliferative rate. A statistically significant difference in the overall survival between the two groups was not seen. Modern Pathology (2010) 23, 235-243; doi:10.1038/modpathol.2009.164; published online 20 November 2009

Keywords: central nervous system; diffuse large B-cell lymphoma; germinal center; immunocompetent

Primary central nervous system lymphoma is defined as the presence of non-Hodgkin's lymphoma

Correspondence: Dr EM Hattab, MD, Department of Pathology and Laboratory Medicine, Indiana University School of Medicine, 350 West 11th Street, CPL 4014, Indianapolis, IN 46202, USA.

E-mail: ehattab@iupui.edu

Received 25 August 2009; revised 8 October 2009; accepted 11

October 2009; published online 20 November 2009 in the brain or spinal cord in the absence of lymph node, bone marrow, or other organ involvement at the time of diagnosis. ${ }^{1}$ It is relatively uncommon, accounting for only $2.7 \%$ of all central nervous system malignancies and $1-2 \%$ of all lymphomas. ${ }^{1,2}$ Immunodeficient patients, including acquired immune deficiency syndrome (AIDS) patients and organ transplant recipients, are at an increased risk of developing primary central nervous system 
lymphomas. ${ }^{1}$ However, even in immunocompetent individuals, the incidence of primary central nervous system lymphoma is increasing. ${ }^{3-5}$ The most common type of primary central nervous system lymphoma is the diffuse large B-cell type ${ }^{6}$ Primary central nervous system diffuse large B-cell lymphoma carries a poor prognosis despite multimodal treatment with methotrexate-based chemotherapy and radiotherapy. Median survival is $17-45$ months in immunocompetent patients, and only 13.5 months in those with AIDS. ${ }^{7}$ Although several clinical factors, including age, performance status, and extent of disease, have been found to be prognostically significant in some studies, ${ }^{8,9}$ a validated method of predicting prognosis in these patients remains to be elucidated.

Recently, diffuse large B-cell lymphomas of lymph node origin as well as those originating in extranodal sites, have been subdivided into three prognostically significant categories by gene expression profiling using cDNA microarrays. ${ }^{10,11}$ These include germinal center, activated B-cell, and type 3 . The type 3 group displays a heterogeneous gene expression pattern, but has a poor outcome similar to the activated B-cell group. These latter two are considered to represent lymphomas of nongerminal center origin. It is now accepted that the clinical outcome of diffuse large B-cell lymphoma of germinal center cell phenotype is better than that of nongerminal center type. In 2004, Hans et $a l^{12}$ successfully used the combined immunohistochemical staining panel of CD10, Bcl-6, and MUM1 to characterize these two prognostic categories. ${ }^{12}$ Accordingly, the germinal center subtype is assigned to those tumors expressing CD10 or CD10 and Bcl-6, whereas the nongerminal center subtype is assigned to those negative for both CD10 and Bcl-6. Lymphomas negative for CD10 but positive for Bcl-6 are further classified based on the expression of MUM1. MUM1 positive cases are assigned to the nongerminal center subtype and MUM1 negative tumors to the germinal center subtype. Several studies have investigated the validity of using this stratification scheme in other extranodal diffuse large B-cell lymphomas; ${ }^{13-18}$ however, it remains unclear whether primary central nervous system diffuse large B-cell lymphomas can be subdivided into these same two prognostic groups.
In this study, we retrospectively investigated the immunostaining profile of 31 well-documented primary central nervous system diffuse large B-cell lymphomas using the same methods as described by Hans et al. ${ }^{12}$ In addition, the proliferative activity was assessed semiquantitatively using Ki-67 antibody. Correlation with follow-up data was performed using the Kaplan-Meier survival curve. Dependent on the availability of the tissue, we evaluated the rearrangements of MYC/IGH and IGH/ BCL2 genes using fluorescence in situ hybridization (FISH).

\section{Materials and methods}

The archival files of Indiana University Medical Center in Indianapolis, Indiana, and Wayne State University affiliated hospitals in Detroit, Michigan, were searched from 2000 to 2006 for primary central nervous system lymphoma of the diffuse large B-cell type. Thirty-one cases that had material sufficient to perform the intended immunohistochemical stains were retrieved. All cases were reviewed and the diagnosis of primary central nervous system diffuse large B-cell lymphoma was confirmed. Those cases exhibiting Burkitt or Burkitt-like morphology were excluded. Primary central nervous system lymphoma was defined as the presence of a brain or spinal cord lesion in the absence of lymph node, bone marrow, or other organ involvement at the time of diagnosis.

\section{Immunohistochemistry}

Formalin-fixed, paraffin-embedded tissue blocks were cut in $4-6 \mu \mathrm{m}$ sections. The sections were then deparaffinized, rehydrated, blocked with 3\% hydrogen peroxide, and appropriately retrieved. A panel of standard well-controlled antibodies was then applied using the modified avidin-biotin method for detection (Table 1). Tonsillar tissue was used as a control.

For each immunostain, the percentage of positive cells was estimated for each of the 10 high power fields evaluated, and an average calculated. Immunostaining for CD10, Bcl-6, and MUM1 was considered positive if $>30 \%$ of the tumor cells were

Table 1 A detailed list of the antibodies used in this study

\begin{tabular}{lllll}
\hline Antibody & Clone & Company & Primary incubation method & Concentration \\
\hline LCA & 2B11+PD7/26 & Dako Inc, Carpinteria, CA & Per Autostainer Link software & RTU \\
CD20 & L 265 & Ventana Medical Systems, Inc, Tuscon, AZ & Benchmark for 16 min & RTU \\
Bcl-2 & 124 & Dako Inc, Carpinteria, CA & Autostainer, 30 min at RT & RTU \\
CD 10 & 56 C6 & Novocastra Laboratories Ltd, Norwell, MA & Manual for 2h at RT & $1: 20$ \\
Bcl-6 & GI191E/A8 & Ventana Medical Systems, Inc, Tuscon, AZ & Benchmark for 32 min & RTU \\
MUM1 & MuM1p & Dako Inc, Carpinteria, CA & Manual overnight at $4{ }^{\circ} \mathrm{C}$ & $1: 20$ \\
KI-67 & K-2 & Ventana Medical Systems, Inc, Tuscon, AZ & Benchmark for 16 min & RTU \\
\hline
\end{tabular}

RT: room temperature; RTU: ready to use. 
immunoreactive. Only nuclear staining was considered positive for MUM1, Bcl-6, and Ki-67. Subclassification was carried out as described earlier according to the schema proposed by Hans et al. ${ }^{12}$ Available clinical and follow-up data were retrieved and tabulated (Table 2).

\section{Fluorescence In Situ Hybridization}

Formalin-fixed, paraffin-embedded, $4 \mu \mathrm{m}$ tissue sections were placed on positively charged glass slides. The tissue sections were baked at $65^{\circ} \mathrm{C}$ for a minimum of $2 \mathrm{~h}$ and deparaffinized in xylene followed by dehydration in $100 \%$ ethanol. Pretreatment of the tissue included exposure to $0.2 \mathrm{~N} \mathrm{HCl}$, distilled water and $2 \times$ SSC at room temperature followed by immersion for $30 \mathrm{~min}$ in a $1 \mathrm{M}$ sodium thiocyanate solution heated to $80^{\circ} \mathrm{C}$. The tissue was digested with a solution of protease and $0.01 \mathrm{~N} \mathrm{HCl}$ warmed to $37^{\circ} \mathrm{C}$ for between $30 \mathrm{~s}$ and $6 \mathrm{~min}$ and rinsed in $2 \times$ SSC.

$M Y C / I G H$ and IGH/BCL2 probe sets manufactured by Abbott Molecular/Vysis (Des Plaines, IL) were used. Both probe sets are structured as dual color/ dual fusion probes to detect translocations by rearrangement of green to red signals and red to green signals, creating yellow (fusion) signals on each derivative chromosome. In the $M Y C / I G H$ probe set, $M Y C$ is labeled with SpectrumOrange and $I G H$ with SpectrumGreen. A probe that hybridizes to the centromere (D8Z2) and labeled with SpectrumAqua was also used. In the $I G H / B C L 2$ probe set, $I G H$ is labeled with SpectrumGreen and BCL2 with SpectrumOrange. In a normal nucleus, two orange and two green signals will be present. In a cell with a translocation, one orange, one green, and two fusion signals are observed. Each probe was validated to confirm location and signal pattern by hybridizing to cells from five normal males and analyzing 20 metaphase cells and 50 interphase nuclei for each.

Each probe was denatured at $75^{\circ} \mathrm{C}$ for $5 \mathrm{~min}$ and kept at $-20^{\circ} \mathrm{C}$ until use. The tissue was denatured by exposure to a denaturation solution composed of $70 \%$ formamide and $2 \times$ SSC at $72^{\circ} \mathrm{C}$ for $11 \mathrm{~min}$ and then dehydrated in a cold graduated alcohol series of 70,85 , and $100 \%$ ethanol. The probe was applied to the tissue and secured with a glass coverslip and rubber cement. The slides were then placed in a humidified chamber and incubated at $37^{\circ} \mathrm{C}$ overnight. Following hybridization, the slides were washed in a solution of $2 \times$ SSC and $3 \%$ NP40 warmed to $73.5^{\circ} \mathrm{C}$, air dried, and exposed to a DAPI and antifade solution.

FISH analysis of the signal pattern was performed by analyzing 100 nuclei in the area of interest with a Leitz DMR fluorescence microscope equipped with both dual and triple pass filters to detect red and green signals and a DAPI background. An aqua filter was also used for examining the $M Y C / I G H$ probe set. The scoring criteria included analysis of only single nuclei with distinct nuclear borders and avoidance of overlapped cells. The presence of at least one green and one red signal/cell was required for analysis.

\section{Results}

The patients' ages ranged from 13 to 81 years old, with a mean age at presentation of 62 years and a median of 64 years. The only child in this series, a 13-year-old, was immunocompotent and had no unusual pertinent history. Sixty-five percent (20/31) of the patients were female, and $94 \%$ (29/31) were Caucasian. The majority of the lymphomas were supratentorial, with only two cases being infratentorial and one case occurring in the spinal cord. None of our patients were known to be HIV positive. One patient had a 10-year history of an orthotopic liver transplant. The patients were collected from two institutions and over a wide time range, and therefore information about their therapy was not accessible for all cases. However, many were treated with high-dose methotrexate and concomitant steroids. As expected, all cases showed diffuse infiltration, often in a sheet-like pattern, and replacement of the brain parenchyma by large lymphoid cells. Characteristic microscopic findings, including perivascular cuffing of tumor cells, large pleomorphic nuclei with prominent nucleoli (Figure 1a), and areas of geographic necrosis (Figure 1b), were seen. The tumor cells showed diffuse, intense CD20 expression (Figure 1c) but were negative for CD3. The liver transplant patient's lymphoma displayed EBV immunopositivity consistent with monomorphic posttransplant lymphoproliferative disorder of diffuse large B-cell lymphoma type.

Of the 31 cases examined, $26(84 \%)$ were of the nongerminal center subtype (Figures 2a-c). Specifically, none of these cases were positive for CD10, 21 showed focal Bcl-6 immunostaining, and 25 were MUM1 positive. The one case with MUM1 negative staining was also CD10 and Bcl-6 negative and therefore was categorized as nongerminal center type. Bcl-2 was positive in $70 \%$ of cases. The Ki-67 proliferative index in these 26 cases ranged from 30 to $90 \%$ (mean, 69\%) (Figure 2d). Five cases were categorized as germinal center subtype (Figures $2 \mathrm{e}-\mathrm{g})$. Four of the five cases were CD10 positive, and the fifth was CD10 negative but Bcl-6 positive and MUM1 negative. They had a Ki-67 index ranging between 70 and $90 \%$ (mean, 78\%) (Figure 2h).

The follow-up intervals ranged from 13.3 to 144.2 months, with a median follow-up interval of 54.3 months. Seven of 31 patients were alive at last follow-up. A Kaplan-Meier survival analysis indicated that there was not a statistically significant difference in survival between germinal center and nongerminal center subtypes (Log-Rank $\chi^{2}=0.18$, $\mathrm{df}=1, \quad P=0.67$ ) (Figure 3 ). The median overall survival times $(95 \% \mathrm{CI})$ were 2.5 months $(0.0-6.6)$ 
Table 2 Summary of the clinical and immunohistochemical findings of 31 primary CNS lymphomas of the diffuse large B-cell type

\begin{tabular}{|c|c|c|c|c|c|c|c|c|c|c|c|c|c|c|c|c|}
\hline Case & Age & Sex 1 & Race I & $L C A$ & $D 20$ & $B c l-2$ & D10 & Bcl-6 & MUM1 & \% Ki-67 & $\begin{array}{l}G C \\
\text { status }\end{array}$ & $\begin{array}{l}\text { Chromosome } 8 \\
(\text { FISH })\end{array}$ & $\begin{array}{l}\text { MYC } \\
(\text { FISH) }\end{array}$ & IGH (FISH) & BCL2 (FISH) & $\begin{array}{l}\text { Follow-up } \\
\text { (months) }\end{array}$ \\
\hline 1 & 13 & $\mathrm{~F}$ & B & + & + & - & + & + & - & 90 & GC & Insufficient cells & Insufficient cells & Insufficient cells & Insufficient cells & AFOD (76.3) \\
\hline 2 & 74 & $\mathrm{~F}$ & W & + & + & + & + & + & - & 70 & GC & Normal & Trisomy & Dual fusion of IGH and BCL2 & & DOD (0.43) \\
\hline 3 & 77 & $\mathrm{M}$ & $\mathrm{W}$ & + & + & + & - & + & - & 70 & GC & Normal & Normal & Dual fusion of IGH and BCL2 & & DOD (0.6) \\
\hline 4 & 52 & $\mathrm{~F}$ & $\mathrm{~B}$ & + & + & + & + & + & + & 90 & GC & NA & NA & NA & NA & AFOD (47.3) \\
\hline 5 & 67 & $\mathrm{~F}$ & W & + & + & + & + & + & + & 70 & GC & Normal & Normal & Normal & Normal & DOD (2.5) \\
\hline 6 & 60 & $\mathrm{~F}$ & W & + & + & + & - & + & + & 30 & NGC & Normal & Normal & Normal & Amplification (3-12) & DOD (6.5) \\
\hline 7 & 62 & $\mathrm{M}$ & $\mathrm{W}$ & + & + & - & - & + & + & 60 & NGC & Normal & Normal & Trisomy & Normal & DOD (0.3) \\
\hline 8 & 49 & $\mathrm{~F}$ & W & + & + & - & - & - & - & 30 & NGC & NA & NA & NA & NA & DOD (7.9) \\
\hline 9 & 68 & $\mathrm{~F}$ & $\mathrm{~W}$ & + & + & + & - & - & + & 40 & NGC & NA & NA & NA & NA & DOD (30.8) \\
\hline 10 & 75 & $\mathrm{~F}$ & $\mathrm{~W}$ & + & + & + & - & + & + & 90 & NGC & Normal & Normal & Normal & Normal & DOD (16.9) \\
\hline 11 & 40 & $\mathrm{M}$ & $\mathrm{W}$ & + & + & - & - & - & + & 90 & NGC & Normal & Normal & Normal & Normal & AFOD (76.9) \\
\hline 12 & 66 & $\mathrm{~F}$ & $\mathrm{~W}$ & + & + & + & - & + & + & 70 & NGC & Normal & Normal & Normal & Normal & DOD (5.5) \\
\hline 13 & 76 & $\mathrm{~F}$ & W & + & + & - & - & + & + & 90 & NGC & NA & NA & NA & NA & DOD (1.2) \\
\hline 14 & 68 & $\mathrm{M}$ & W & + & + & + & - & + & + & 90 & NGC & Normal & Normal & Normal & Normal & DOD (7.8) \\
\hline 15 & 64 & $\mathrm{~F}$ & $\mathrm{~W}$ & + & + & + & - & - & + & 60 & NGC & Normal & Normal & Normal & Normal & DOD (1.2) \\
\hline 16 & 62 & $\mathrm{~F}$ & W & + & + & - & - & + & + & 90 & NGC & NA & NA & NA & NA & DOD (0.7) \\
\hline 17 & 66 & $\mathrm{~F}$ & $\mathrm{~W}$ & + & + & NA & - & + & + & 90 & NGC & Insufficient cells & Insufficient cells & Insufficient cells & Insufficient cells & AFOD (30.1) \\
\hline 18 & 60 & $\mathrm{M}$ & $\mathrm{W}$ & + & + & + & - & + & + & 50 & NGC & Normal & Normal & Normal & Trisomy/tetrasomy & DOD (7.4) \\
\hline 19 & 63 & $\mathrm{~F}$ & $\mathrm{~W}$ & + & + & + & - & + & + & 60 & NGC & Normal & Normal & Normal & Normal & DOD (19.5) \\
\hline 20 & 42 & $\mathrm{~F}$ & $\mathrm{~W}$ & + & + & - & - & + & + & 90 & NGC & Trisomy/tetrasomy & Trisomy/tetrasomy & Trisomy/pentasomy & Trisomy/tetrasomy & DOD (13.5) \\
\hline 21 & 70 & $\mathrm{M}$ & W & + & + & + & - & + & + & 75 & NGC & Normal & Normal & Normal & Normal & DOD (11) \\
\hline 22 & 47 & $\mathrm{~F}$ & $\mathrm{~W}$ & + & + & + & - & + & + & 30 & NGC & Normal & Normal & Normal & Trisomy/tetrasomy & DOD (3.3) \\
\hline 23 & 79 & $\mathrm{~F}$ & $\mathrm{~W}$ & + & + & + & - & + & + & 70 & NGC & Normal & Normal & Normal & Normal & DOD (2.2) \\
\hline 24 & 53 & $\mathrm{~F}$ & W & + & + & - & - & + & + & 90 & NGC & Normal & Normal & Normal & Normal & DOD (3.1) \\
\hline 25 & 80 & $\mathrm{M}$ & W & + & + & + & - & + & + & 75 & NGC & Normal & Normal & Normal & Normal & DOD (1.4) \\
\hline 26 & 58 & $\mathrm{M}$ & $\mathrm{W}$ & + & + & + & - & + & + & 80 & NGC & Normal & Normal & Normal & Normal & AFOD (25.3) \\
\hline 27 & 47 & $\mathrm{~F}$ & $\mathrm{~W}$ & + & + & - & - & + & + & 50 & NGC & NA & NA & NA & NA & DOD (3.9) \\
\hline 28 & 51 & $\mathrm{M}$ & $\mathrm{W}$ & + & + & + & - & + & + & 60 & NGC & NA & NA & NA & NA & DOD (9.6) \\
\hline 29 & 81 & $\mathrm{M}$ & W & + & + & + & - & - & + & 90 & NGC & NA & NA & NA & NA & DOD (1.6) \\
\hline 30 & 73 & $\mathrm{~F}$ & W & + & + & + & - & + & + & 60 & NGC & Normal & Normal & Normal & Normal & AFOD (24.5) \\
\hline 31 & 65 & $\mathrm{M}$ & W & + & + & + & - & + & + & 90 & NGC & Normal & Normal & Monosomy & Trisomy & AFOD (13.3) \\
\hline
\end{tabular}

AFOD: alive free of disease; NA: not available; DOD: died of disease; GC: germinal center; NGC: non-germinal center.

.

.



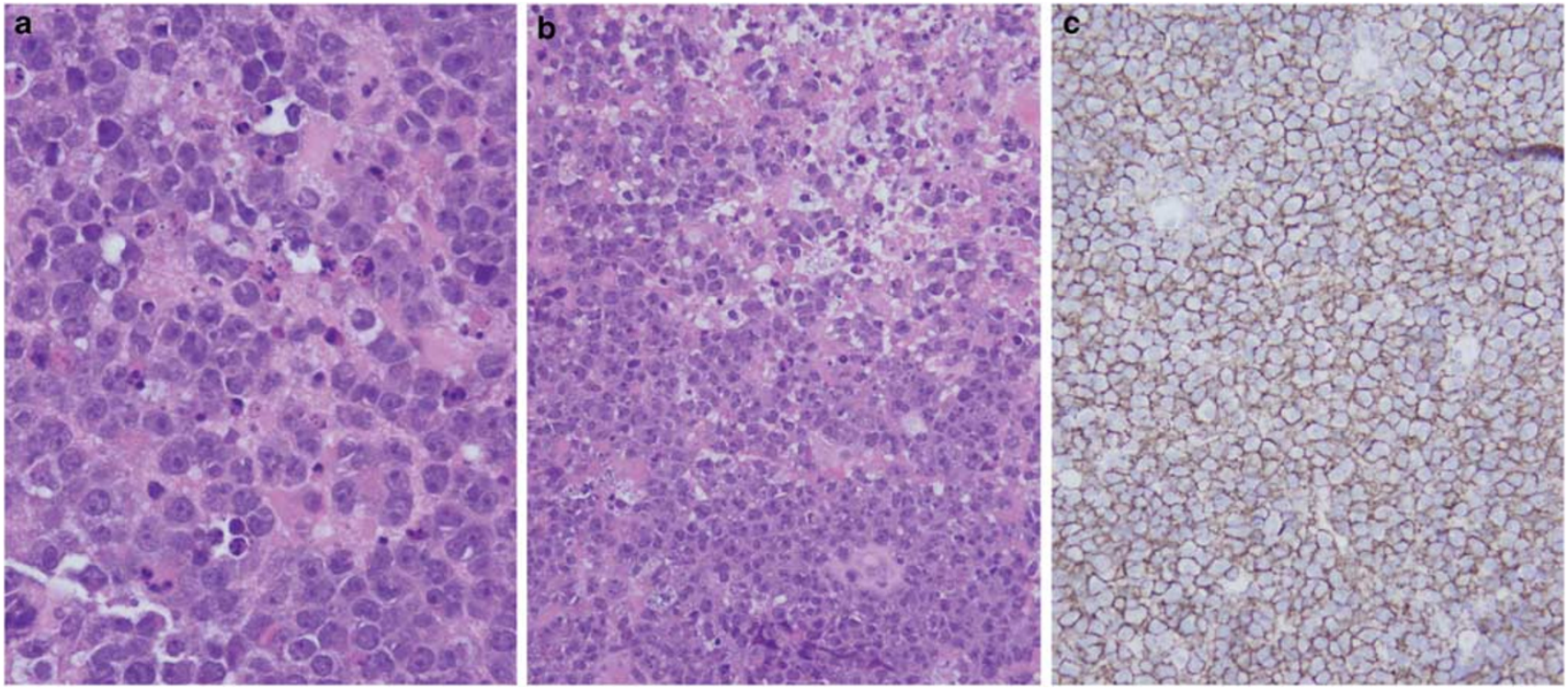

Figure 1 The primary central nervous system lymphomas in this study displayed characteristic large B-cell morphology, including large pleomorphic nuclei with prominent nucleoli (a) and geographic necrosis (b), as well as diffuse CD20 positivity (c).

and 7.4 months (4.4-10.4) for the germinal center and nongerminal subtypes, respectively. The 5-year survival rates were $40.0 \%$ for the germinal center and $10.8 \%$ for the nongerminal subtypes. The overall 5 -year survival rate was $18.6 \%$.

Mean and median survivals for the 26 Bcl-6 positive cases were 12.8 and 6.95 months, respectively, whereas for the five Bcl-6 negative cases, mean and median survivals were 23.7 and 7.9 months, respectively. Statistical analysis was not performed.

FISH for $M Y C / I G H$ and IGH/BCL2 was successfully performed in 21 cases (Table 1). The majority of cases (62\%) showed normal IGH, MYC, and BCL2 signals. Two cases, both of germinal center origin as determined by immunohistochemistry, demonstrated $\mathrm{t}(14 ; 18)(\mathrm{q} 32 ; \mathrm{q} 21)$. In one additional case, the BCL2 gene was amplified. Those cases showing rearrangement/amplification of the BCL2 gene also showed Bcl-2 protein expression by immunohistochemistry. However, many cases expressed Bcl-2 by immunohistochemistry and did not show abnormalities in the $B C L-2$ gene, signifying poor correlation between Bcl-2 immunohistochemistry and BCL2 gene status by FISH. In addition, several cases showed aberrant copy numbers of studied genes (Table 2, monosomy to pentasomy for $M Y C, I G H$, and BCL2). None of the cases showed the isolated $M Y C / I G H$ rearrangement characteristic of Burkitt's lymphoma, confirming the morphological and immunohistochemical subclassification of our cases as diffuse large B-cell lymphoma. No cases showed 'double-hit' translocations. A Kaplan-Meier overall survival analysis was performed on the two groups: Group 1 (G1) being the eight cases (38\%) with abnormalities by FISH analysis and Group 2 (G2) being the 13 patients (62\%) with normal FISH analysis. Statistical analysis showed a trend toward shorter survival for those patients with FISH abnormalities, but it lacked statistical significance $\left(\log -\operatorname{Rank} \chi^{2}=1.763, \mathrm{df}=1, P=0.148\right)$ (Figure 4).

\section{Discussion}

In 2004, Hans et $a l^{12}$ used a panel of immunohistochemical stains, including CD10, Bcl-6, and MUM1, to categorize diffuse large B-cell lymphomas into two groups, as differentiated earlier by gene expression arrays: germinal center and nongerminal center. They also found that the germinal center group had a much better prognosis (76\% 5-year overall survival) compared with the nongerminal center diffuse large B-cell lymphoma (34\%). ${ }^{12}$ Subsequent studies have confirmed these results in nodal and noncentral nervous system, extranodal diffuse large B-cell lymphomas.

In this study, we analyzed the immunoprofile of 30 cases of primary central nervous system diffuse large B-cell lymphoma from immunocompetent patients and one case from an immunocompromised patient. The majority ( $84 \%$ ) of the cases examined were of nongerminal center origin. This finding is consistent with several other earlier published studies. Braaten et al studied 33 patients with primary central nervous system diffuse large B-cell lymphoma. Seventy-nine percent of the cases were positive for Bcl-6, 19\% were positive for CD10, and $97 \%$ were positive for MUM1. ${ }^{19}$ Even though the authors did not classify the lymphomas into germinal center and nongerminal center subtypes using the schema proposed by Hans et al, it is evident that the majority of cases are of nongerminal center origin. Lin et $a l^{20}$ studied 51 patients with primary central nervous system diffuse large B-cell 

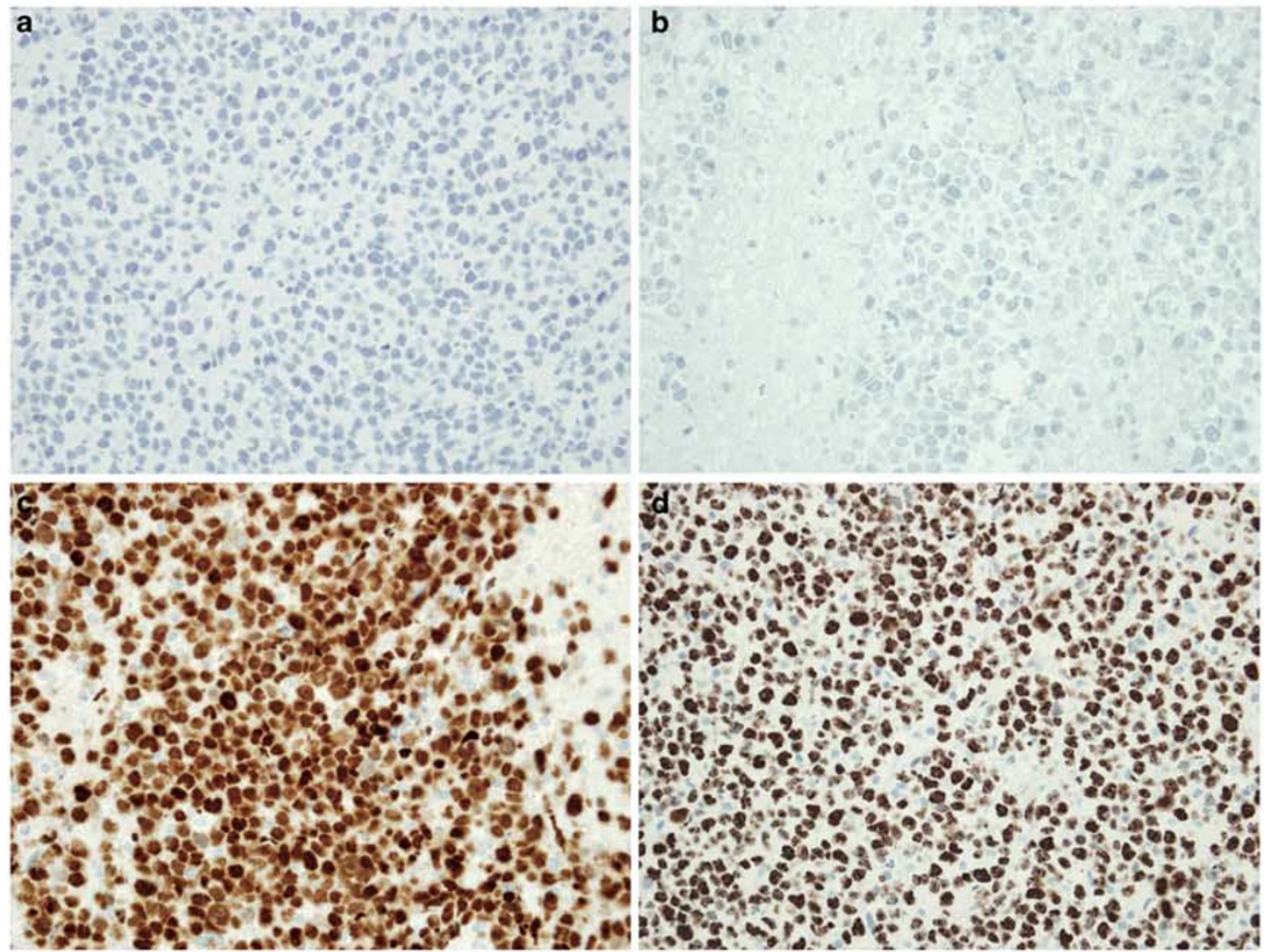

der.
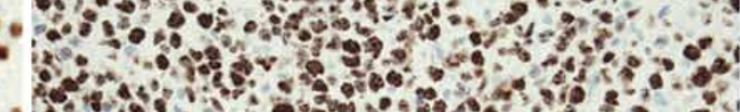

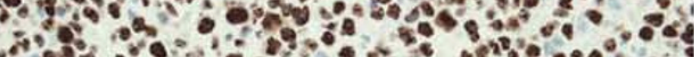

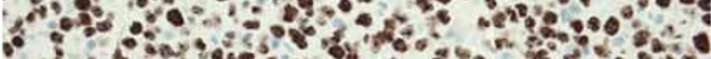
1.6

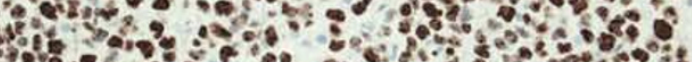

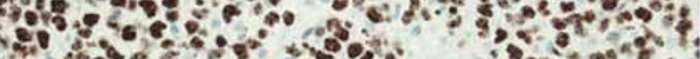

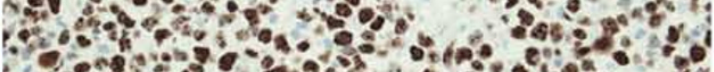

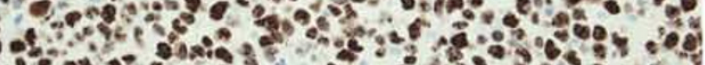

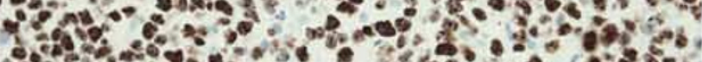

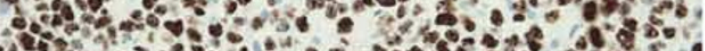
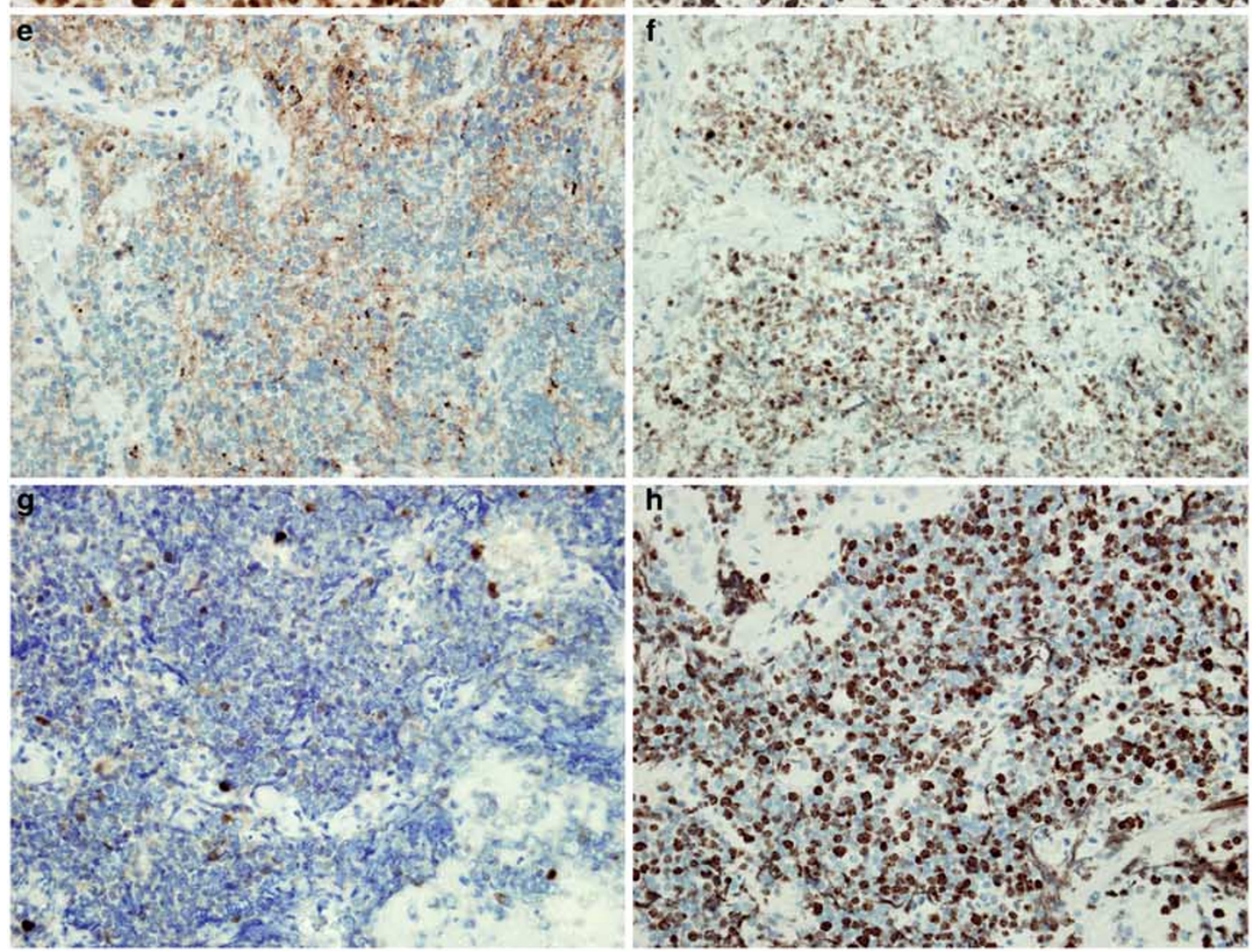

Figure 2 The immunohistochemical panel for case 6 (top rows) was CD10 negative (a), Bcl-6 negative (b), MUM1 positive (c), and displayed high proliferative activity as labeled by Ki-67 (d). This case belonged to the nongerminal center subtype and similar findings were found for the other 25 cases of this subtype. The immunohistochemical panel for case 1 (bottom rows) was CD10 positive (e), Bcl-6 positive (f), MUM1 negative (g), and showed high proliferative activity as labeled by Ki-67 (h). This case belonged to the germinal center subtype. 


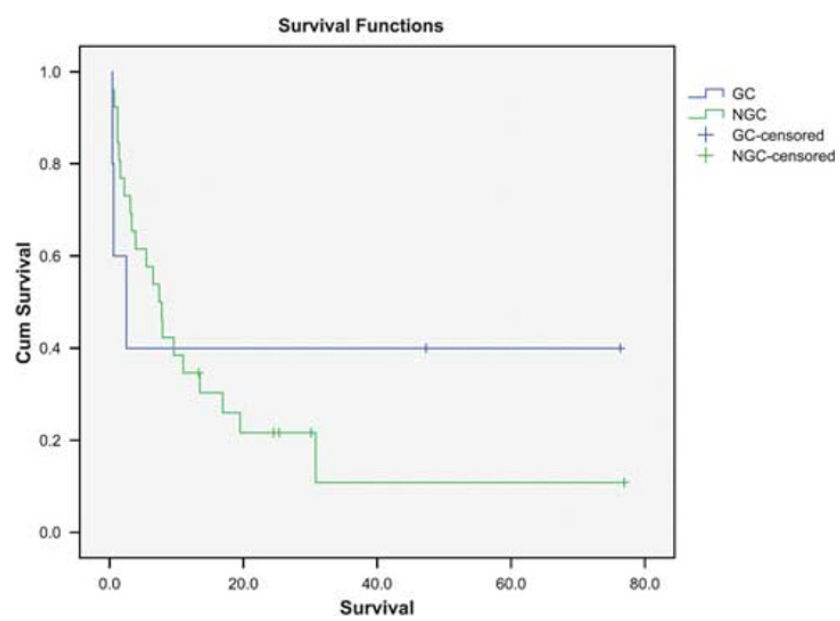

Figure 3 Kaplan-Meier curve showed no statistically significant difference in survival between the germinal center (GC) and nongerminal center (NGC) groups.

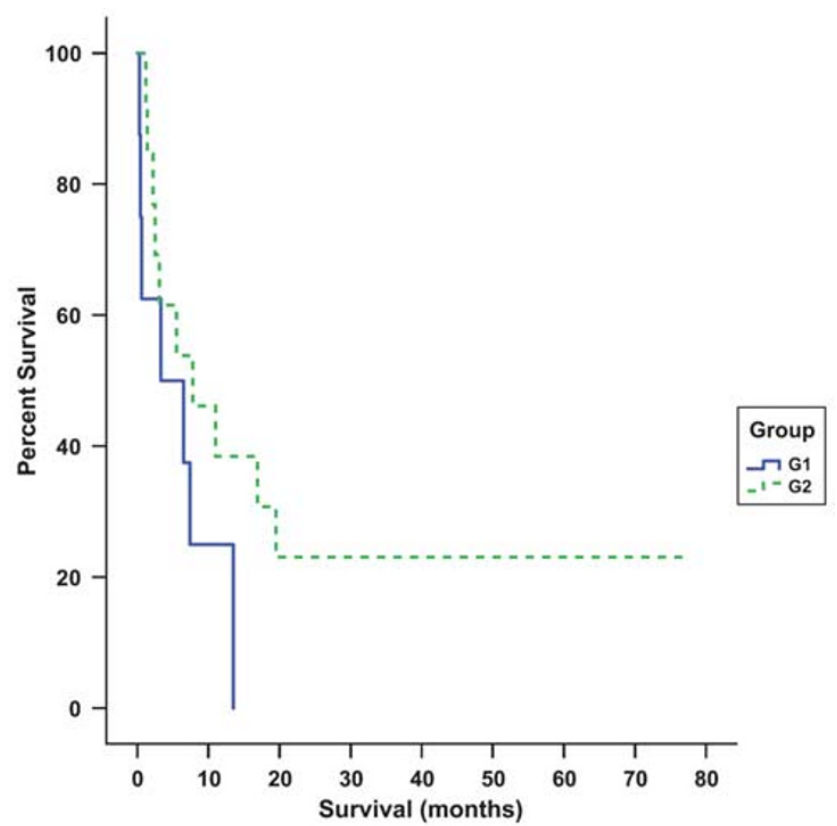

Figure 4 Kaplan-Meier curve showed no statistically significant difference in survival between the group with FISH abnormalities (G1) and those (G2) without such abnormalities.

lymphoma and found $40 / 51(78 \%)$ to be of the nongerminal center subtype and $11 / 51(22 \%)$ of the germinal center subtype. In addition, CamilleriBroët et $a l^{21}$ analyzed 83 patients with primary central nervous system diffuse large B-cell lymphoma using the same immunomarkers applied in our study and found $96.3 \%$ of the cases to be of the nongerminal center subtype.

Although earlier studies have also categorized primary central nervous system diffuse large B-cell lymphoma into germinal center and nongerminal center subtypes, to our knowledge, our study is the first to correlate these subtypes with survival out- comes. Although nodal diffuse large B-cell lymphomas of the nongerminal center type are thought to be prognostically less favorable than those of the germinal center type, our initial findings in primary central nervous system diffuse large B-cell lymphomas showed no statistically significant difference in survival between the two groups. It may be postulated that the inherently high-grade nature of these neoplasms and their poorer prognosis compared with noncentral nervous system extranodal diffuse large B-cell lymphomas probably contributes to the lack of significant difference in clinical outcome between these two groups. In addition, the relatively small number of germinal center subtype cases (five) may have also contributed.

Several earlier studies have examined the prognostic significance of Bcl-6 expression in primary central nervous system diffuse large B-cell lymphomas and reported conflicting results. Braaten et al ${ }^{19}$ and Lin et $a l^{20}$ both found Bcl-6 expression to be an independent predictor of improved survival, whereas Chang et $a l^{22}$ found that patients with Bcl-6 expression had a poorer overall survival and Camilleri-Broët et $a l^{21}$ found no difference in survival between the two groups. Our results indicate a trend toward decreased survival for those patients whose tumors were Bcl-6 positive.

We also found that all of the primary central nervous system diffuse large B-cell lymphomas studied herein exhibited high proliferative activity, regardless of their germinal center status. As stated above, in general, primary central nervous system lymphomas tend to have a poorer prognosis than nodal lymphomas, which may be partly explained by their tendency to have a high proliferative activity and be of the nongerminal center subtype.

The literature shows a clear association between immunosuppression and primary central nervous system lymphoma, and it has long been thought that most primary central nervous system lymphomas occur in immunosuppressed patients. ${ }^{1,6}$ This common conception may have evolved because of the sharp increase in incidence of primary central nervous system lymphomas during the early AIDS epidemic. However, with the advent of highly active antiretroviral therapy, the incidence of central nervous system lymphomas in AIDS patients has decreased dramatically. ${ }^{6}$ Currently, primary central nervous system lymphomas may occur just as, or perhaps more, frequently in immunocompetent patients than in immunocompromised ones. Indeed, only $1 / 31$ patient $(3 \%)$ in this series was known to be immunosuppressed. None of the patients discussed herein were known to have HIV infection, but one patient (\#9) did have a history of orthotopic liver transplantation and was receiving long-term immunosuppressive therapy. It should be pointed out, however, that many of the patients in our study were not retrospectively tested as most were viewed as belonging to a relatively low-risk population, and their HIV status was essentially unknown. 
Our FISH analysis using probes for $M Y C, I G H$, and $B C L 2$ genes indicates abnormalities in $38 \%$ of the cases. Earlier studies of primary central nervous system lymphomas in immunocompetent hosts revealed similar rates of $M Y C / I G H$ and IGH/BCL2 abnormalities. ${ }^{23-25}$ Interestingly, both in our study and in earlier reports, the majority of primary central nervous system lymphomas show strong expression of Bcl-2 and C-MYC, indicating that overexpression at protein and mRNA levels is poorly correlated with the rearrangements of $B C L 2$ and $C-M Y C$ genes. ${ }^{25,26}$ Thirty-three percent $(7 / 21)$ of cases showed additional signals for IGH, BCL2, and $C-M Y C$. These most likely represent additional gene copies due to trisomy or tetrasomy; however, the rearrangement to an unknown partner cannot be entirely excluded. Numerical chromosomal abnormalities, known as aneuploidy, occur frequently in diffuse large B-cell lymphomas and have been suggested to arise due to centrosome defects. ${ }^{27}$

Some studies have suggested that patients who harbor such abnormalities have a shorter overall survival. ${ }^{23,25}$ In our study, despite the apparent trend toward shorter survival in these patients, the difference was not statistically significant. The lack of statistical significance may be related to the small number of our patient sample. Of the five germinal center subtype cases, two showed abnormalities by FISH analysis. Of the 26 nongerminal center subtype cases, six showed anomalies. As the number of cases in the former group was small, we were unable to draw any comparative conclusions between the two groups.

In summary, most of the primary central nervous system diffuse large B-cell lymphomas in our study were of the nongerminal center subtype. Although studies of nodal and noncentral nervous system extranodal diffuse large B-cell lymphomas have found the nongerminal center subtype to be prognostically less favorable, we found no statistically significant difference in survival between the two groups. We also found that abnormalities involving the $M Y C / I G H$ and $I G H / B C L 2$ genes are relatively frequent.

\section{Acknowledgement}

Presented, in part, at the 82nd annual meeting of the American Association of Neuropathologists, San Francisco, California, 10-15 September 2006.

\section{Disclosure/conflict of interest}

The authors declare no conflict of interest.

\section{References}

1 Bhagavathi S, Wilson JD. Primary central nervous system lymphoma. Arch Pathol Lab Med 2008;132: 1830-1834.
2 Powari M, Radotra B, Das A, et al. A study of primary central nervous system lymphoma in northern India. Surg Neurol 2002;57:113-116.

3 Maher EA, Fine HA. Primary CNS lymphoma. Semin Oncol 1999;26:346-356.

4 Miller DC, Hochberg FH, Harris NL, et al. Pathology with clinical correlations of primary central nervous system non-Hodgkin's lymphoma. The Massachusetts General Hospital experience 1958-1989. Cancer 1994; 74:1383-1397.

5 Cote TR, Manns A, Hardy CR, et al. Epidemiology of brain lymphoma among people with or without acquired immunodeficiency syndrome. AIDS/Cancer Study Group. J Natl Cancer Inst 1996;88:675-679.

6 Deckert M, Paulus W. Malignant lymphomas In: Louis DN, Ohgaki H, Wiestler OD, Cavenee WK (eds). WHO Classification of Tumours of the Central Nervous System, 4th edn, International Agency for Research on Cancer: Lyon, 2007, pp 188-192.

7 Chamberlain MC. Long survival in patients with acquired immune deficiency syndrome-related primary central nervous system lymphoma. Cancer 1994;73:1728-1730.

8 Bessell EM, Graus F, Lopez-Guillermo A, et al. Primary non-Hodgkin's lymphoma of the CNS treated with CHOD/BVAM or BVAM chemotherapy before radiotherapy: long-term survival and prognostic factors [see comment]. Int J Radiat Oncol Biol Phys 2004;59: 501-508.

9 Ferreri AJM, Blay J-Y, Reni M, et al. Prognostic scoring system for primary CNS lymphomas: the International Extranodal Lymphoma Study Group experience. J Clin Oncol 2003;21:266-272.

10 Alizadeh AA, Eisen MB, Davis RE, et al. Distinct types of diffuse large B-cell lymphoma identified by gene expression profiling [see comment]. Nature 2000;403:503-511.

11 Rosenwald A, Wright G, Chan WC, et al. The use of molecular profiling to predict survival after chemotherapy for diffuse large-B-cell lymphoma [see comment]. N Engl J Med 2002;346:1937-1947.

12 Hans CP, Weisenburger DD, Greiner TC, et al. Confirmation of the molecular classification of diffuse large B-cell lymphoma by immunohistochemistry using a tissue microarray. Blood 2004;103: 275-282.

13 Al-Abbadi MA, Hattab EM, Tarawneh MS, et al. Primary testicular diffuse large B-cell lymphoma belongs to the nongerminal center B-cell-like subgroup: a study of 18 cases. Mod Pathol 2006;19: 1521-1527.

14 Berglund M, Thunberg U, Amini R-M, et al. Evaluation of immunophenotype in diffuse large B-cell lymphoma and its impact on prognosis. Mod Pathol 2005;18:1113-1120.

15 Chang C-C, McClintock S, Cleveland RP, et al. Immunohistochemical expression patterns of germinal center and activation B-cell markers correlate with prognosis in diffuse large B-cell lymphoma. Am J Surg Pathol 2004;28:464-470.

16 Hoffmann C, Tiemann M, Schrader C, et al. AIDSrelated B-cell lymphoma (ARL): correlation of prognosis with differentiation profiles assessed by immunophenotyping. Blood 2005;106:1762-1769.

17 van Imhoff GW, Boerma E-JG, van der Holt B, et al. Prognostic impact of germinal center-associated proteins and chromosomal breakpoints in poor-risk 
diffuse large B-cell lymphoma. J Clin Oncol 2006;24:4135-4142.

18 Yoshida S, Nakamura N, Sasaki Y, et al. Primary breast diffuse large B-cell lymphoma shows a non-germinal center B-cell phenotype. Mod Pathol 2005;18:398-405.

19 Braaten KM, Betensky RA, de Leval L, et al. BCL-6 expression predicts improved survival in patients with primary central nervous system lymphoma. Clin Cancer Res 2003;9:1063-1069.

20 Lin C-H, Kuo K-T, Chuang S-S, et al. Comparison of the expression and prognostic significance of differentiation markers between diffuse large B-cell lymphoma of central nervous system origin and peripheral nodal origin. Clin Cancer Res 2006;12:1152-1156.

21 Camilleri-Broët S, Criniere E, Broet $\mathrm{P}$, et al. A uniform activated B-cell-like immunophenotype might explain the poor prognosis of primary central nervous system lymphomas: analysis of 83 cases. Blood 2006; 107:190-196.

22 Chang C-C, Kampalath B, Schultz C, et al. Expression of p53, c-Myc, or Bcl-6 suggests a poor prognosis in primary central nervous system diffuse large B-cell lymphoma among immunocompetent individuals. Arch Pathol Lab Med 2003;127:208-212.

23 Cady FM, O’Neill BP, Law ME, et al. Del(6)(q22) and BCL6 rearrangements in primary CNS lymphoma are indicators of an aggressive clinical course. J Clin Oncol 2008;26:4814-4819.

24 Nozaki M, Tada M, Mizugaki Y, et al. Expression of oncogenic molecules in primary central nervous system lymphomas in immunocompetent patients. Acta Neuropathol 1998;95:505-510.

25 Rubenstein JL, Fridlyand J, Shen A, et al. Gene expression and angiotropism in primary CNS lymphoma. Blood 2006;107:3716-3723.

26 Cobbers JM, Wolter M, Reifenberger J, et al. Frequent inactivation of CDKN2A and rare mutation of TP53 in PCNSL. Brain Pathol 1998;8:263-276.

27 Kramer A, Schweizer S, Neben K, et al. Centrosome aberrations as a possible mechanism for chromosomal instability in non-Hodgkin's lymphoma. Leukemia 2003;17:2207-2213. 